\title{
Spin-orbital Kondo decoherence by environmental effects in capacitively coupled quantum dots
}

\author{
Sabine Andergassen, ${ }^{1}$ Pascal Simon, ${ }^{2,3}$ Serge Florens, ${ }^{1}$ and Denis Feinberg ${ }^{1}$ \\ ${ }^{1}$ Institut NEEL, Centre National de la Recherche Scientifique and Université Joseph Fourier, BP 166, 38042 Grenoble, France \\ ${ }^{2}$ Laboratoire de Physique et Modélisation des Milieux Condensés, \\ Centre National de la Recherche Scientifique and Université Joseph Fourier, 38042 Grenoble, France \\ ${ }^{3}$ Department of Physics and Astronomy, University of Basel, \\ Klingelbergstrasse 82, CH-4056, Basel, Switzerland
}

(Dated: October 29, 2018)

\begin{abstract}
Strong correlation effects in a capacitively coupled double quantum-dot setup were previously shown to provide the possibility of both entangling spin-charge degrees of freedom and realizing efficient spin-filtering operations by static gate-voltage manipulations. Motivated by the use of such a device for quantum computing, we study the influence of electromagnetic noise on a general spin-orbital Kondo model, and investigate the conditions for observing coherent, unitary transport, crucial to warrant efficient spin manipulations. We find a rich phase diagram, where low-energy properties sensitively depend on the impedance of the external environment and geometric parameters of the system. Relevant energy scales related to the Kondo temperature are also computed in a renormalization-group treatment, allowing to assess the robustness of the device against environmental effects. These are minimized at low bias voltage and for highly symmetric devices, concerning the geometry.
\end{abstract}

PACS numbers: 72.15.Qm,71.10.Pm,72.10.Fk,73.63.Kv

\section{INTRODUCTION}

Controlling and manipulating isolated spins in quantum dots has been the subject of intense research in the last years. One goal of this line of research is the realization of novel spin-based devices which may provide new ways of processing quantum information. An architecture for spin-based quantum computing has been pro-

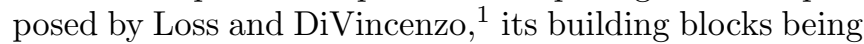
quantum dots made from a two-dimensional electron gas. Recent developments using time-dependent gates led to considerable progress, achieving a single spin qubit control via electron spin resonance in a double-dot (DD) device. 2

Another possibility however consists in realizing single or two-spin operations in a given device, which acts as a logical gate, using static control parameters only, such as gate voltages or constant magnetic fields. Along this direction, complex operations such as the production of entangled electron pairs,$\underline{\underline{3}}$ or spin teleportation $\underline{4}$ were proposed. This scheme, avoiding multiple and synchronized time manipulations, may increase the processing rate and facilitate the integration into more complex devices. A promising approach for manipulating spins in semiconductor nanostructures consists in the use of a gate-controlled spin-orbit (Rashba) coupling, $\underline{\underline{5} \underline{6}}$ Spin precession has been recently revealed in metallic rings with spin-orbit interaction. ${ }^{7}$ Another way of controlling the spin was proposed by two of the authors in a system of two capacitively coupled quantum dots under a magnetic field operating with two extra electrons in the charge sector. This setup implements an entanglement of spin and orbital degrees of freedom by the realization of an artificial spin-orbit coupling, fully tunable by a gate voltage only. When such a DD system is driven into the Kondo regime a spin-orbital Kondo effect occurs, where each spin flip is associated with an orbital flip, i.e. with an electron transfer from one dot to the other through the leads. Owing to the unitary transport that takes place below the Kondo temperature, this property was exploited to propose an efficient (i.e. highconductance) Stern-Gerlach spin filter separating an unpolarized spin current into two polarized ones with opposite magnetization $\stackrel{8}{-}$ Moreover, we demonstrated that such a DD operates as a Stern-Gerlach interferometer (SGI) in presence of a single common lead. A coherent spin precession can be supplied by an Aharonov-Bohm (AB) flux obtained by slightly tilting the in-plane magnetic field,,$\underline{9}$ allowing for a controlled realization of a onequbit phase gate on spin qubits.

Since the efficiency of the above device is directly related to the possibility of cooling the system below the Kondo temperature, and hence of forming a strongcoupling Kondo resonance, the question arises how an external electromagnetic environmental affects the Kondo physics in the case of strongly entangled spin-orbit quantum states. The study of single and double tunnel junctions, as well as of transistors in a noisy environment modeled by an impedance established more than fifteen years ago the mechanism of dynamical Coulomb blockade (DCB) (see Refs. 10,11] for a review). Decoherence effects due to electromagnetic environment have been studied for series,,$\frac{12}{1}$ and paralle 13,14 double dot systems in the sequential regime. The interplay between the Kondo effect and the background charge fluctuations has however not yet been analyzed systematically; the issue was addressed only recently for some specific systems. Previous investigations of the Kondo effect in presence of DCB 
focused on the regime near the charge degeneracy point of a quantum dot coupled to a noisy back gate. 15,16,17,18 In this case a Kondo model for the charge degree of freedom can be derived, including a direct coupling of the charge variable to the dissipative environmental modes. This generates a competition between the Kondo screening of the charge doublet by the electrons and the localization effect due to the ohmic environment. On the other hand, the physics and the transport properties for the conventional Kondo effect in the spin sector under an electromagnetic noise has also been analyzed recently and appears to be more subtle. The case of an ac excitation was treated in Refs. [19 20], while that of an ohmic noise was addressed in Ref. [21]. This study showed that an ohmic resistance of the environment exceeding half the quantum value $R_{K}=h / e^{2}$ induces a suppression of the inter-lead Kondo interactions, without however preventing the formation of a strong-coupling state due to the remaining intra-lead processes. Transport through the device is therefore suppressed in a way similar to the usual DCB, while spin screening can be completely or partially preserved. For an environmental resistance smaller than $R_{K} / 2$ the Kondo effect can normally develop between the localized spin and both leads. However, the fully transparent fixed point with unitary conductance $G=2 e^{2} / h$ is only stable when particle-hole symmetry is maintained by the dot plunger gate voltage. These striking results imply that, even though the Kondo screening of the local spin survives, non-linear transport properties through the device appear in general at lowtemperature, and should be revealed by tuning a strong environmental impedance.

The present study aims first at merging these previously separate analyses of the charge and spin Kondo effects in the presence of an ohmic environment into a detailed study of background charge fluctuations in a Kondo model where spin and orbital degrees of freedom are entangled. The second aspect of this work will highlight how the electromagnetic noise may affect the spinorbital Kondo effect and, as a consequence, reduce the potential performance of logical spin operations.

The paper is structured as follows. In Sec. II we introduce the model and derive the mapping to a general spin-orbital Kondo model characterized by a maximal entanglement of spin and orbital degrees of freedom. In Sec. [II] we present a phenomenological description of the environmental fluctuations using the equivalent circuit theory. This description enables to derive an effective low-energy model that combines both the spin, or equivalently the orbital fluctuations, and the background electromagnetic noise, assumed to be ohmic in the present analysis. In Sec. IV we provide a perturbative renormalization-group ( $\mathrm{RG}$ ) analysis for the low-energy Hamiltonian. In particular, we discuss the decoherence of the spin-orbital Kondo effect induced by the ohmic bath and elaborate the generic low-energy phase diagram, that allows for an interpretation of the complicated flow of the Kondo couplings. Strong renormalization effects on the
Kondo scale may be observed for particular parameters, restricting the use of the device as a high-conductance spin filter. The particular case of a pure orbital Kondo effect obtained in capacitively coupled quantum dots is finally analyzed. We briefly summarize our results in Sec. V.

\section{SPIN-ORBITAL KONDO EFFECT IN A DOUBLE QUANTUM DOT}

In this section we focus on the orbital Kondo effect in the DD. We are particularly interested in the spin splitter or SGI introduced previously 8.9 The typical device we have in mind is depicted in Fig. 1. Nevertheless, this device is rather generic and encompasses other geometries based on capacitively coupled quantum dots where orbital Kondo physics is expected $:^{22,23,24}$

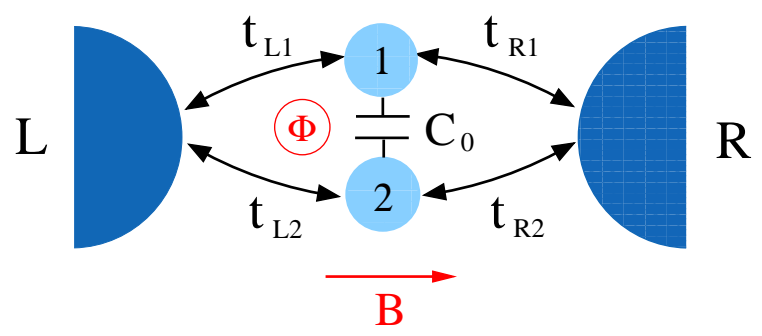

FIG. 1: (Color online) Schematic representation of the proposed setup: two small quantum dots coupled by a capacitance $C_{0}$ and connected to left and right reservoirs. Depending on the choice of the gate voltages, the upper branch filters spin- $\uparrow$ and the lower one spin- $\downarrow$, or vice versa. A magnetic flux $\Phi$ threads the whole device.

\section{A. Derivation of the model}

The number of electrons on a single orbital level in each dot is controlled by two plunger gate voltages via the capacitances $C_{g 1}$ and $C_{g 2}$, and by a coupling capacitance $C_{0}$. The charge states are labeled by $\left(n_{1}, n_{2}\right)$ extra electrons in dot 1 and 2 respectively. Both dots are connected to the same left and right lead with tunneling hopping parameters $t_{L 1 / 2}$ and $t_{R 1 / 2}$ respectively. The regime we consider throughout this paper is characterized by the situation where the low-energy charge states $(1,1)$ and $(0,2)$ are almost degenerate ${ }^{8}$ These correspond to eight spin-charge states, whose degeneracy will however be lifted by Zeeman and orbital splitting as discussed below. For energies lower than the charging energy of the dot $E_{c}=\min (E(0,1)-E(1,1) ; E(1,2)-E(1,1))$ the charge dynamics is restricted to these states only, the states $(0,1)$ and $(1,2)$ occurring as virtual processes. The lowest excited states $(0, \uparrow)$ and $(\uparrow, \uparrow \downarrow)$ are assumed to involve the same charge excitation energy $E_{c}=\frac{e^{2} C_{0}}{4 C\left(C+2 C_{0}\right)}$, 
with $C=C_{L}+C_{R}+C_{g}$ for equal dots and $C_{L / R}$ left $/$ right junction capacitances.

At low energy the isolated DD system is described by ${ }^{22}$

$$
H_{\mathrm{dot}}=-\delta E T^{z}-t T^{x}-g \mu_{B} B S^{z},
$$

where we defined the orbital pseudospin as

$$
T^{z}=\left(n_{1}-n_{2}+1\right) / 2= \pm 1 / 2 .
$$

Here $\delta E=E(0,2)-E(1,1)$ is zero when the two lowest charge states are exactly degenerate. The second term in Eq. (1) represents a small parasitic tunneling amplitude between the $\operatorname{dots}^{8}$ and the last term is the Zeeman splitting in presence of a local magnetic field applied in the $z$ direction. We assume that the Zeeman energy is large enough so that the total spin-charge states of the setup amount to the two degenerate ground states $(\uparrow, \uparrow)$ and $(0, \uparrow \downarrow)$. Note that a large level spacing $\delta \epsilon$, or equivalently small quantum dots are necessary to neglect triplet states $, 26,27$ When this condition is fulfilled, the total spin $S^{z}=S_{1}^{z}+S_{2}^{z}$ is maximally entangled with the orbital pseudospin since $S^{z}=T^{z}$. As a consequence a spin flip always involves an orbital pseudo-spin flip. Note that quantum coherence between spin and orbital degrees of freedom has been put forward in some SU(4) Kondo effect. $22,28,29,30$

Therefore, this setup provides a realization of an artificial and fully controlled local spin-orbit coupling. Besides a stationary spin- $\uparrow$ electron in the lower dot, the lowenergy Kondo screening of the spins by the reservoirs involves spin- $\uparrow$ electrons in the upper path and spin- $\downarrow$ electrons in the lower one and vise versa, corresponding to a maximal entanglement of spin and orbital (or dot) degrees of freedom. This property has been applied in the proposal of an efficient spin splitter in Ref. [8] and in the realization of single spin operations. $\frac{9}{}$

The full Hamiltonian is

$$
H=H_{\text {dot }}+H_{\text {leads }}+H_{\text {tun }},
$$

where the leads are described by the Hamiltonian

$$
H_{\text {leads }}=\sum_{k, \gamma, \sigma} \varepsilon_{k} c_{k, \gamma, \sigma}^{\dagger} c_{k, \gamma, \sigma}
$$

$c_{k, \gamma, \sigma}^{\dagger}$ creates an electron with energy $\varepsilon_{k}$ and spin $\sigma$ in the lead $\gamma=L, R$ (the Zeeman splitting in the reservoirs is negligible) 26 The tunneling terms between the leads and the dots are given by

$$
H_{\mathrm{tun}}=\sum_{k, \gamma, j, \sigma}\left(t_{\gamma j} c_{k, \gamma, \sigma}^{\dagger} d_{j, \sigma}+\text { H.c. }\right) \text {, }
$$

where $d_{j, \sigma}$ destroys an electron with spin $\sigma$ in $\operatorname{dot} j=$ 1,2 . Note that the orbital pseudospin is expressed as $T^{z}=S^{z}=\left(d_{1, \uparrow}^{\dagger} d_{1, \uparrow}-d_{2, \downarrow}^{\dagger} d_{2, \downarrow}\right) / 2$. In order to determine the effective coupling between the DD and the leads, we consider virtual excitations of the two excited states $(1,2)$ and $(0,1)$ due to tunneling processes between the leads and the dots. Using second-order perturbation theory ${ }^{9}$ in the tunneling amplitudes the Kondo Hamiltonian $H_{K}$ reads

$$
\begin{aligned}
H_{K}=\frac{1}{2} & {\left[T^{z}\left(J_{L L}^{z \uparrow} \psi_{L \uparrow}^{\dagger} \psi_{L \uparrow}-J_{L L}^{z \downarrow} \psi_{L \downarrow}^{\dagger} \psi_{L \downarrow}+J_{R R}^{z \uparrow} \psi_{R \uparrow}^{\dagger} \psi_{R \uparrow}-J_{R R}^{z \downarrow} \psi_{R \downarrow}^{\dagger} \psi_{R \downarrow}\right)\right.} \\
& +T^{z}\left(e^{-i \alpha / 2} J_{L R}^{z \uparrow} \psi_{L \uparrow}^{\dagger} \psi_{R \uparrow}-e^{i \alpha / 2} J_{L R}^{z \downarrow} \psi_{L \downarrow}^{\dagger} \psi_{R \downarrow}+\text { H.c. }\right) \\
& \left.+T^{-}\left(J_{L L}^{\perp} e^{-i \alpha / 2} \psi_{L \uparrow}^{\dagger} \psi_{L \downarrow}+J_{R R}^{\perp} e^{i \alpha / 2} \psi_{R \uparrow}^{\dagger} \psi_{R \downarrow}+J_{L R}^{\perp} \psi_{L \uparrow}^{\dagger} \psi_{R \downarrow}+J_{R L}^{\perp} \psi_{R \uparrow}^{\dagger} \psi_{L \downarrow}\right)+\text { H.c. }\right],
\end{aligned}
$$

where $\psi_{\gamma \sigma}=\sum_{\mathbf{k}} c_{\mathbf{k}, \gamma, \sigma} e^{i \mathbf{k} \mathbf{R}_{\gamma \sigma}}$, and $\alpha=2 \pi \Phi / \Phi_{0}$ with the flux quantum $\Phi_{0}$ is the AB phase within the loop. The phase factor $e^{i \mathbf{k R}_{\gamma \sigma}}$ with $\mathbf{R}_{\gamma \uparrow}=\mathbf{R}_{\gamma \mathbf{1}}$ and $\mathbf{R}_{\gamma \downarrow}=\mathbf{R}_{\gamma \mathbf{2}}$ takes into account the distance $\mathbf{R}_{\gamma \uparrow}-\mathbf{R}_{\gamma \downarrow}$ between tunnel junctions and distinguishes the different orbital states in the leads. The operators $T^{+}$and $T^{-}$flip the orbital pseudo-spin. We introduced several Kondo couplings

$$
J_{\gamma \gamma^{\prime}}^{\perp} \approx \frac{t_{\gamma 1} t_{\gamma^{\prime} 2}}{E_{c}} \text { and } J_{\gamma \gamma^{\prime}}^{z \uparrow / \downarrow} \approx \frac{t_{\gamma 1 / 2} t_{\gamma^{\prime} 1 / 2}}{E_{c}}
$$

where $\gamma, \gamma^{\prime}=L, R$.

\section{B. Magnetic field effects}

We note that the $J_{\gamma \gamma^{\prime}}^{z \uparrow / \downarrow}$ Kondo couplings are spin dependent, except for $t_{\gamma / \gamma^{\prime} 1} \simeq t_{\gamma / \gamma^{\prime} 2}$. A similar situation appears for a small quantum dot in the conventional Kondo regime connected to spin-polarized leads. The spin polarization induces a splitting of the Kondo resonance which can be compensated by an external magnetic field. ${ }^{31}$ In the present case, due to the entanglement of orbital and spin degrees of freedom, the compensation for such a geometric asymmetry is achieved with an orbital field, i.e. by fine-tuning the dot gate voltages $V_{g 1}$ and $V_{g 2}$. If not stated differently, we therefore assume $t_{\gamma / \gamma^{\prime} 1} \simeq t_{\gamma / \gamma^{\prime} 2}$ in the following. 
We can rule out the phase in the Hamiltonian (6) by defining the new basis $\tilde{\psi}_{L / R \uparrow}=e^{ \pm i \alpha / 4} \psi_{L / R \uparrow}$ and $\tilde{\psi}_{L / R \downarrow}=e^{\mp i \alpha / 4} \psi_{L / R \downarrow}$. In this spin-rotated basis the Kondo Hamiltonian takes the conventional form

$$
H_{K}=\sum_{\gamma, \gamma^{\prime}} \sum_{\sigma \sigma^{\prime}} J_{\gamma \gamma^{\prime}} \tilde{\psi}_{\gamma \sigma}^{\dagger} \vec{T} \cdot \vec{\tau}_{\sigma \sigma^{\prime}} \tilde{\psi}_{\gamma^{\prime} \sigma^{\prime}} .
$$

The disappearance of the AB phase indicates the absence of interference in the present geometry under specific conditions discussed below. Indeed, electrons with a spin- $\uparrow$ travel through the upper dot whereas electrons with spin$\downarrow$ take the lower one. In this regime, corresponding to the unitary limit, the spin $\vec{S}$, or equivalently the pseudo-spin $\vec{T}$, is completely screened and a spin singlet is formed with the left and right electrodes.

\section{INCLUSION OF ENVIRONMENTAL FLUCTUATIONS}

A main source of decoherence relies in the circuit electromagnetic fluctuations, coupling to tunneling events to and from each of the two dots. As a consequence the orbital/spin doublet does not fulfill anymore the condition of perfect degeneracy and the quantity $\delta E$ in Eq. (1) dynamically fluctuates around zero. Here we focus on decoherence effects induced by a strong ohmic environment.

\section{A. Equivalent circuit}

A convenient starting point employs an equivalent circuit representation of the SGI following Refs. [10] and [32]. It contains two single-electron transistors in parallel, coupled by a capacitance. We model the electromagnetic fluctuations by introducing an impedance $Z$ closing the equivalent circuit as depicted in Fig. 2. We neglect the dot gate voltage fluctuations as the dot sizes are very small and hence $C_{g j} \ll C_{\gamma j}$. Starting from a more complicated equivalent circuit, they can be taken into account. 32

Here the environment couples to virtual charge fluctuations as in cotunneling processes. DCB of inelastic

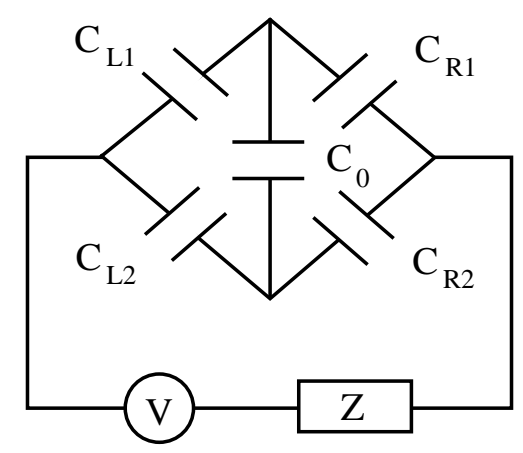

FIG. 2: Schematic representation of the equivalent circuit. cotunneling through two junctions in series was treated in Ref. [33], while high-order processes coupling tunneling in two parallel junctions were investigated in Ref. [34].

At the Hamiltonian level, electromagnetic fluctuations can be included by the following transformation of the tunneling amplitudes

$$
t_{\gamma j} \rightarrow t_{\gamma j} e^{i \varphi_{\gamma j}}
$$

in Eq. (5) and subsequently in Eq. (6) through the Kondo couplings defined by Eqs. (7), see Sec. IIIB. The phases $\varphi_{\gamma j}$ are related to the voltage fluctuations $\delta V_{\gamma j}$ felt by an electron during a tunneling event through the junction

$$
\varphi_{\gamma j}(t)=\frac{e}{\hbar} \int_{-\infty}^{t} \delta V_{\gamma j}\left(t^{\prime}\right) d t^{\prime}
$$

The phases $\varphi_{\gamma j}$ are conjugated of the charges $Q_{\gamma j}$ on the junction capacitance $C_{\gamma j}$ such that $\left[\varphi_{\gamma j}, Q_{\gamma j}\right]=i e$ on each dot. As the phases $\varphi_{\gamma j}$ originate from the same electromagnetic bath, they are clearly not independent. Following the general procedure of Ref. [10], the above phases can be expressed in terms of three phases $\varphi, \psi_{1}, \psi_{2}$, where $\varphi$ is the phase conjugated of the total charge $Q$ seen by the impedance, and $\psi_{1 / 2}$ is the phase conjugated of the charge in dot $1 / 2$ respectively. In the present analysis we consider the experimentally most relevant case of an ohmic impedance $Z(0)=R$, and define the dimensionless parameter $r=R / R_{K}$.

We first need to relate the phases $\varphi_{\gamma j}$ to $\varphi$. The simplest technique involves the equivalent circuit shown in Fig. 1, determining the impedance $Z_{\gamma j}$ and the voltage $V_{\gamma j}$ seen by the junction $\gamma j$ during a tunneling event. $Z_{\gamma j}$ and $V_{\gamma j}$ are obtained in a straightforward manner using successive Thevenin-Norton transformations as described in Ref. [10]. This yields $Z_{\gamma j}=\kappa_{\gamma j}^{2} Z$ and $V_{\gamma j}=\kappa_{\gamma j} V$, where the constant $\kappa_{\gamma j}$ depends on the various capacitances via 


$$
\kappa_{L / R, 1 / 2}=\frac{\left(C_{L, 2 / 1}+C_{R, 2 / 1}\right) C_{R / L, 1 / 2}+C_{0}\left(C_{R / L, 1}+C_{R / L, 2}\right)}{\left(C_{L 1}+C_{R 1}\right)\left(C_{L 2}+C_{R 2}\right)+C_{0}\left(C_{L 1}+C_{R 1}+C_{L 2}+C_{R 2}\right)} .
$$

We therefore infer that

$$
\varphi_{\gamma j}=\kappa_{\gamma j} \varphi+a_{\gamma j} \psi_{1}+b_{\gamma j} \psi_{2}
$$

where the coefficients $a_{\gamma j}, b_{\gamma j}$ are determined by the circuit theory. The phases $\psi_{i}$ related to the charge on the dots have purely imaginary correlators at long time and can be discarded $\stackrel{10}{\underline{n}}$ Another systematic way to find these results is to compute directly all the correlators $\left\langle\varphi_{\gamma j}(t) \varphi_{\gamma^{\prime} j^{\prime}}(0)\right\rangle$ through the involved transimpedances. Note that $\kappa_{L, 1 / 2}+\kappa_{R, 1 / 2}=1$. Indeed, electron transfer processes from one lead to the other without spin flip affect the phase $\varphi$ directly and the system behaves as a double junction in series ${ }^{33,34}$

\section{B. Effective Kondo Hamiltonian}

The derivation of an effective Kondo Hamiltonian instead involves cotunneling in presence of electromagnetic fluctuations as in Ref. [21]. We therefore proceed similarly through a generalized Schrieffer-Wolff transformation involving the excitations of the bath degrees of freedom. We resort again to the quasi-elastic approximation used in Ref. [21], assuming that the energy exchanged with the environment during the cotunneling process is small compared to the charging energy $E_{c}$. Note that similar approximations have been used to treat a single quantum dot in the Kondo regime under an external ac field. $\underline{\underline{19}}$ Under this approximation, the Kondo couplings defined in Eqs. (7) are simply dressed by the corresponding phase operators and become

$$
\begin{aligned}
J_{\gamma \gamma^{\prime}}^{\perp} & \rightarrow \widetilde{J}_{\gamma \gamma^{\prime}}^{\perp}=e^{i\left(\varphi_{\gamma 1}-\varphi_{\gamma^{\prime} 2}\right)} J_{\gamma \gamma^{\prime}}^{\perp} \\
J_{\gamma \gamma^{\prime}}^{z \uparrow / \downarrow} & \rightarrow \widetilde{J}_{\gamma \gamma^{\prime}}^{z \uparrow / \downarrow}=e^{i\left(\varphi_{\gamma 1 / 2}-\varphi_{\gamma^{\prime} 1 / 2}\right)} J_{\gamma \gamma^{\prime}}^{z \uparrow / \downarrow} .
\end{aligned}
$$

Therefore, the effective low-energy Hamiltonian which encompasses both spin-orbit entanglement and environmental fluctuations has exactly the same form as the Kondo Hamiltonian derived in Eq. (6), except that the Kondo couplings acquire now a dynamical phase, i.e. $J_{i} \rightarrow \widetilde{J}_{i}$

We point out the essential property that an originally spin-isotropic Kondo Hamiltonian becomes generally anisotropic by including these charge fluctuations.
This impurity problem is thus more complicated than the conventional Kondo problem because of these dynamical anisotropies, and will be investigated in the following.

IV. DECOHERENCE OF THE SPIN-ORBITAL KONDO EFFECT

In this section, we want to study at which degree the spin-orbital Kondo effect is robust with respect to the electromagnetic background fluctuations. In the lowenergy limit, we can expect at least two phases: i) one where the spin-orbital Kondo effect fully develops, with a complete screening of the impurity spin below the Kondo temperature; ii) another one where the spin is completely localized in one of the $\uparrow$ or $\downarrow$ states. In fact, as we will see, and as emphasized previously 21 , transport properties in the Kondo phase also strongly depend on the strength of the dissipation.

\section{A. Renormalization-group equations}

For symmetric tunneling amplitudes, where the indices 1 and 2 as well as the distinction between $J^{\perp}$ and $J^{z}$ becomes superfluous, we recover the results derived in Ref. [21]. This symmetry is not preserved in general for dynamical phases $\varphi_{\gamma j}$ leading to ten Kondo couplings with $J_{L R}^{z \uparrow / \downarrow}=J_{R L}^{z \uparrow / \downarrow}$. In order to derive the RG flow equations, it may be more convenient to introduce the dimensionless Kondo couplings $\lambda_{i}=\rho \widetilde{J}_{i}$. For simplicity we first focus on the case of equal initial couplings, i.e. $\lambda_{\gamma \gamma^{\prime}}^{z \uparrow /, \perp}=\lambda_{0}$ for all $\gamma, \gamma^{\prime}$; hence $\lambda_{L L}^{z \uparrow / \downarrow}=\lambda_{R R}^{z \downarrow / \uparrow}$ and $\lambda_{L L}^{\perp}=\lambda_{R R}^{\perp}$. The respective tunneling amplitudes $t_{\gamma, \gamma^{\prime}}$ are equal for all $\gamma, \gamma^{\prime}$ according to Eqs. (7), while the capacitances of the equivalent circuit are assumed as independent parameters. Nevertheless, the analysis of this particular case allows for an intuitive understanding of the dynamical emergence of different phases. Moreover, the corresponding phase diagram turns out to contain the generic low-energy physics. The corresponding flow equations read 


$$
\begin{aligned}
\frac{d \lambda_{L L}^{z \uparrow}}{d \ln \Lambda} & =-\frac{1}{2}\left[\left(\lambda_{L L}^{z \uparrow}\right)^{2}+\left(\lambda_{L L}^{\perp}\right)^{2}+\left(\lambda_{L R}^{z \uparrow / \downarrow}\right)^{2}+\left(\lambda_{L R}^{\perp}\right)^{2}\right] \\
\frac{d \lambda_{L L}^{z \downarrow}}{d \ln \Lambda} & =-\frac{1}{2}\left[\left(\lambda_{L L}^{z \downarrow}\right)^{2}+\left(\lambda_{L L}^{\perp}\right)^{2}+\left(\lambda_{L R}^{z \uparrow / \downarrow}\right)^{2}+\left(\lambda_{R L}^{\perp}\right)^{2}\right] \\
\frac{d \lambda_{L L}^{\perp}}{d \ln \Lambda} & =\beta^{2} r \lambda_{L L}^{\perp}-\frac{1}{2}\left[\lambda_{L L}^{\perp}\left(\lambda_{L L}^{z \uparrow}+\lambda_{L L}^{z \downarrow}\right)+\lambda_{L R}^{z \uparrow / \downarrow}\left(\lambda_{L R}^{\perp}+\lambda_{R L}^{\perp}\right)\right] \\
\frac{d \lambda_{L R}^{z \uparrow / \downarrow}}{d \ln \Lambda} & =r \lambda_{L R}^{z \uparrow / \downarrow}-\frac{1}{2}\left[\lambda_{L R}^{z \uparrow / \downarrow}\left(\lambda_{L L}^{z \uparrow}+\lambda_{L L}^{z \downarrow}\right)+\lambda_{L L}^{\perp}\left(\lambda_{L R}^{\perp}+\lambda_{R L}^{\perp}\right)\right] \\
\frac{d \lambda_{L R}^{\perp}}{d \ln \Lambda} & =(1-\beta)^{2} r \lambda_{L R}^{\perp}-\left(\lambda_{L R}^{\perp} \lambda_{L L}^{z \uparrow}+\lambda_{L L}^{\perp} \lambda_{L R}^{z \uparrow / \downarrow}\right) \\
\frac{d \lambda_{R}^{\perp} L}{d \ln \Lambda} & =(1+\beta)^{2} r \lambda_{R L}^{\perp}-\left(\lambda_{R L}^{\perp} \lambda_{L L}^{z \downarrow}+\lambda_{L L}^{\perp} \lambda_{L R}^{z \uparrow / \downarrow}\right)
\end{aligned}
$$

These equations explicitely depend on the dimensionless resistance $r=R / R_{K}$ of the external circuit, and on the capacitances through the prefactor $\beta=\kappa_{L 2}-\kappa_{L 1}$ de- scribing the capacitance asymmetry of the system. The explicit dependence on the capacitances of the equivalent circuit of Fig. 2 is given by

$$
\beta=\frac{C_{L 1} C_{R 2}-C_{R 1} C_{L 2}}{\left(C_{L 1}+C_{R 1}\right)\left(C_{L 2}+C_{R 2}\right)+C_{0}\left(C_{L 1}+C_{R 1}+C_{L 2}+C_{R 2}\right)},
$$

with $\beta \in[-1,1]$. We focus on $\beta>0$, which breaks the symmetry of the system, as a sign change corresponds to an exchange of $L \leftrightarrow R$ lead or $1 \leftrightarrow 2$ dot.

The general case for arbitrary initial couplings, taking into account geometric effects on the capacitances, is discussed subsequently.

\section{B. Symmetric tunneling amplitudes}

\section{Phase diagram}

As detailed below, the analysis of the flow equations (14) for various values of $\beta$ and $r$ allows to determine the phase diagram of the system depicted in Fig. 3 .

This phase diagram is rather complex, and five different regions within two distinct phases can be identified. At small $r$, the region I corresponds to the expected spinorbital Kondo phase, while for larger $r$, the regions IIIIV, characterized by variations of the Kondo fixed point as discussed subsequently, emerge from a crossover region II. The region V corresponds to a different phase and arises for both large values of the dissipation parameter $r$ and intermediate capacitance asymmetry $\beta$; it corresponds to a regime where spin flips are forbidden by the environment (localization). The proximity to this phase is unfavorable to the spin-orbital Kondo effect, and thus detrimental to the spin filtering efficiency.
Before studying the generic case for arbitrary $\beta$ we first discuss the limit $\beta=0$ corresponding to symmetric capacitance configurations. We further extend the analysis to finite $0<\beta<1$, and finally address the limit of maximal capacitance asymmetry for $\beta \lesssim 1$. Realizations of the limiting cases for a simplified parametrization are shown in Fig. 4,

\section{Symmetric capacitances: $\beta=0$}

For $\beta=0$ the system (14) reduces to two equations describing the weak-coupling behavior of $\lambda_{L L}$ and $\lambda_{L R}$

$$
\begin{aligned}
\frac{d \lambda_{L L}}{d \log \Lambda} & =-\lambda_{L L}^{2}-\lambda_{L R}^{2} \\
\frac{d \lambda_{L R}}{d \log \Lambda} & =r \lambda_{L R}-2 \lambda_{L R} \lambda_{L L},
\end{aligned}
$$

as examined in Ref. 21]. The inter-lead or "backscattering" Kondo coupling $\lambda_{L R}$ involves environmental effects via the dynamical phase $\varphi(t)$, while the intra-lead coupling $\lambda_{L L}$ instead is not dressed by phase fluctuations within the quasi-elastic approximation.

The results for the flow equations (16) for different dissipation strengths $r$ are shown in Fig. 5

With increasing $r$ a systematic decrease of the Kondo temperature $T_{K}$ is observed, marking a delay for the 


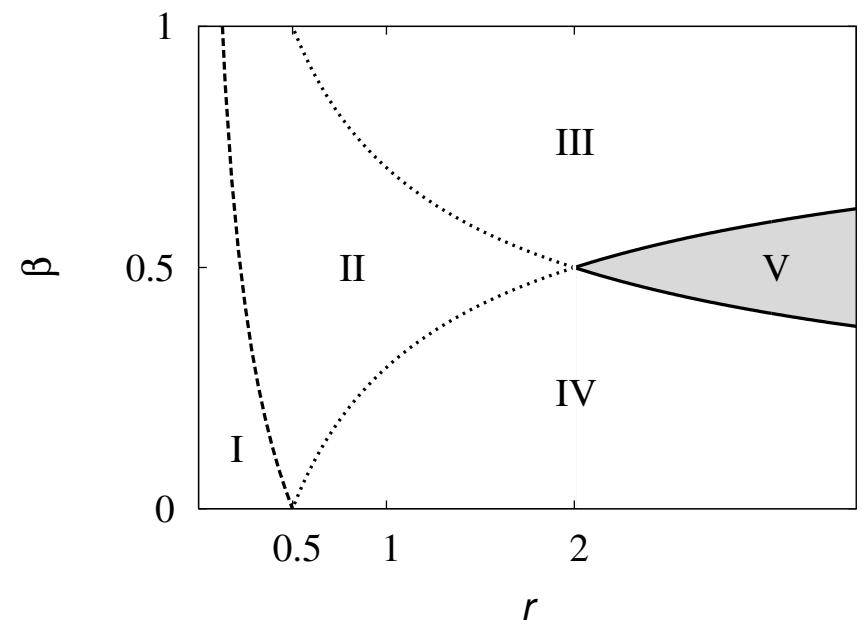

FIG. 3: Phase diagram as a function of dissipation $r$ and capacitance asymmetry parameter $\beta$, without geometric asymmetry in the tunneling amplitudes. The region I corresponds to a robust single-channel spin-orbital Kondo effect, and II to a complex crossover regime that leads to two different Kondo dominated regions: III displays a purely orbital Kondo effect, while IV is associated with a two-channel Kondo effect (for balanced couplings). Finally $\mathrm{V}$ is a distinct phase where the spin is fully localized (analogous to unscreening in the ferromagnetic Kondo problem). The solid line indicates a phase transition, whereas the dashed lines represent a smooth evolution into the crossover region II. See text for details.

onset of the strong-coupling regime. Moreover, the appearance of different strong-coupling fixed points can be identified. For a small environmental resistance compared to the quantum value $R_{K}$ a non-ohmic behavior characterized by DCB prevails. A more detailed analysis shows that this result extends also to unbalanced initial couplings $\stackrel{21}{=}$ For values of $R$ comparable to $R_{K}$ the dissipative term in Eq. (16b) controls the RG flow, inducing a suppression of the inter-lead coupling $\lambda_{L R}$ and therefore of the coherent charge transfer between left and right lead. Due to the remaining intra-lead processes the cou-

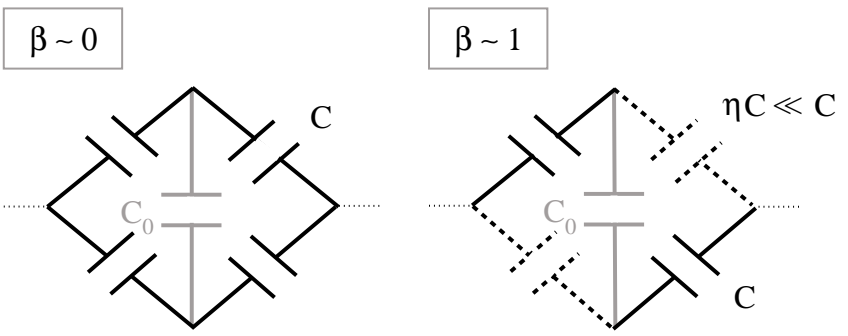

FIG. 4: Realizations of symmetric capacitance configurations for $\beta=0$ and maximal capacitance asymmetry for $\beta \lesssim 1$, with capacitances $C$ (solid lines) and $\eta C$ (dashed lines). Note that $\beta=0$ includes any configuration of the capacitances symmetric under the exchange of the dot and/or lead indices independently of $C_{0}$, whereas $\beta \lesssim 1$ is obtained for $\eta \ll 1$ and $C_{0} \ll C$.

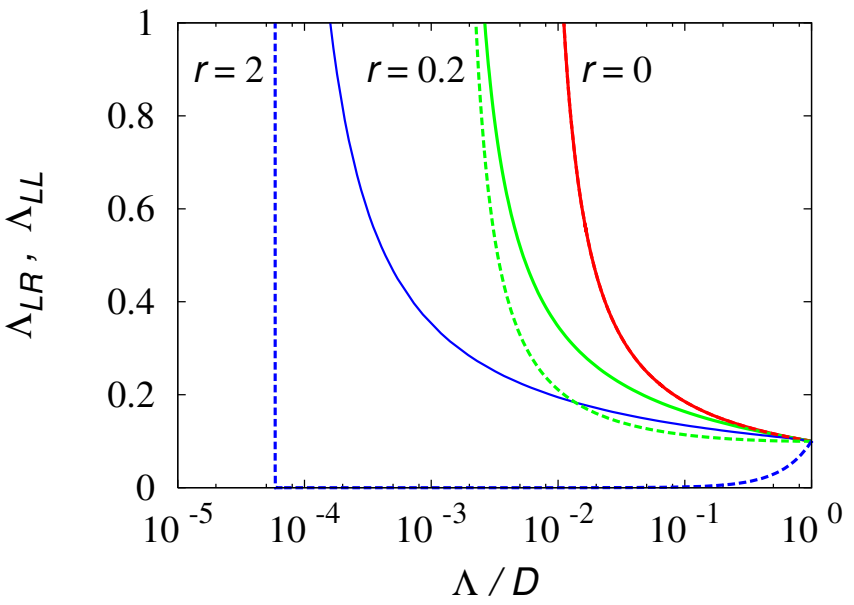

FIG. 5: (Color online) Flow of the Kondo couplings $\lambda_{L L}$ (full line) and $\lambda_{L R}$ (dashed line) with the running cutoff $\Lambda$, according to Eqs. (16), for different values of $r$ in the symmetric case $\beta=0$. The initial values of the Kondo couplings at the initial scale $\Lambda_{0}=D$ (which corresponds to the electronic bandwidth) are taken here as $\lambda_{L L}=\lambda_{L R}=0.1$.

pling $\lambda_{L L}$ (and $\lambda_{R R}$ as well) still renormalize to strong coupling according to Eq. (16a). This corresponds to a phase where the spin flip processes are still coherent but charge transfer between the left and right leads is incoherent. More challenging from the experimental point of view, a large dissipation drives the electron tunneling to zero and a non-ohmic regime develops, characterized in the spin sector by a Kondo effect in the most strongly coupled electrode. For the symmetric case with $\lambda_{L L}=\lambda_{R R}$ a two-channel Kondo fixed point is stabilized by DCB. These results are confirmed by a strongcoupling analysis from the low-energy equivalence of a single Kondo quantum dot in an ohmic environment described by Eqs. (16), and the problem of a $S=1 / 2$ magnetic impurity coupled to Luttinger liquid leads. Two distinctive regimes arise depending on $r>r_{c}$ or $r<r_{c}$, with the critical value $r_{c}=1 / 2$. These two phases, associated with single and two-channel Kondo respectively, correspond in the phase diagram depicted in Fig. 3 to the phases I and IV. In particular, phase IV is characterized by anomalous low-temperature transport properties, accessible in a strong-coupling analysis $\stackrel{21}{\underline{\underline{2}}}$ Let us now analyze the general asymmetric case $\beta \neq 0$.

\section{Asymmetric capacitances: $\beta>0$}

We numerically solved the flow equations (14) for various values of $\beta$ and $r$. Fig. [6 shows results for several Kondo couplings as a function of dissipation $r$ and asymmetry parameter $\beta$. The value for all the initial Kondo couplings at scale $\Lambda_{0}=D$ was chosen to be $\lambda_{i}=0.1$, and the presented data correspond to a set of running couplings $\lambda_{i}$ at the intermediate scale $\Lambda^{*}=\sqrt{D T_{K}}$ for better readability, where $T_{K}$ is defined by the strongest 

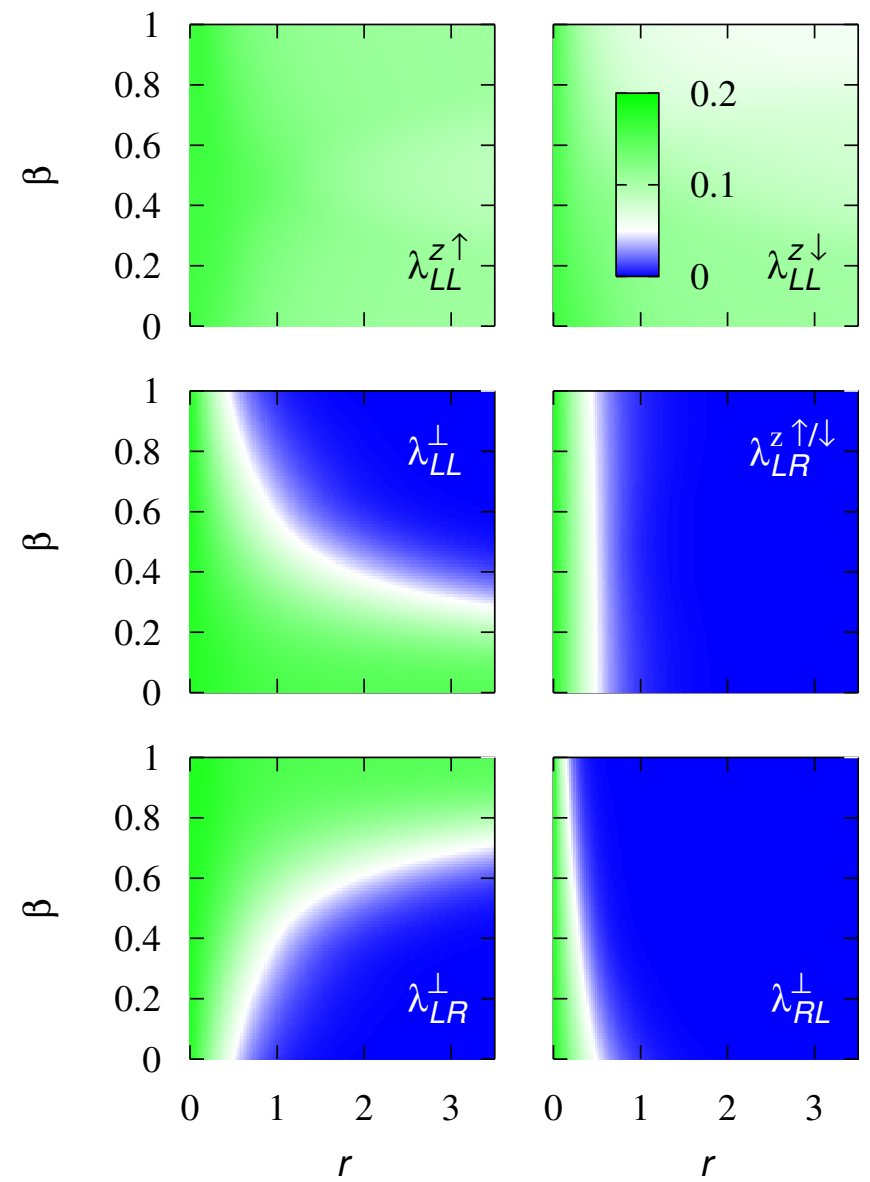

FIG. 6: (Color online) Typical value of the renormalized Kondo couplings $\lambda_{i}$ according to Eqs. (14) with equal initial couplings. These are displayed as a function of dissipation $r$ and asymmetry parameter $\beta$. See text for details.

divergent coupling, at the value $\lambda_{L L}^{z \uparrow}\left(\Lambda=T_{K}\right)=10$. This three-dimensional representation of the running coupling constants allows us to immediately read which of them are driven to strong coupling or are irrelevant in the lowenergy limit.

From the different panels we can directly identify several distinct regimes: $\beta \gtrsim 0, \beta \lesssim 1$ and $\beta \simeq 1 / 2$.

\section{Case $\beta \gtrsim 0$.}

For small values of $\beta$ the physical picture is expected to be controlled by the proximity to the $\beta=0$ line, discussed in Sec. IVB2 In this case, the first three equations of the system (14), corresponding to the intra-lead processes, reduce to Eq. (16a), and the other inter-lead couplings follow Eq. (16b). The dissipation affects more strongly the latter ones and drives them irrelevant for large values of $r$, as shown on the fourth to sixth lower panels of Fig. 6. Nevertheless, one can see that these are not driven irrelevant simultaneously for given values of $\beta$ and $r$. Suppose, we fix for example $\beta \approx 0.3$, we see that for moderate values of $r \simeq 0.8, \lambda_{R L}^{\perp}$ and $\lambda_{L R}^{z \uparrow / \downarrow}$ are driven irrelevant whereas $\lambda_{L R}^{\perp}$ is still going to strong coupling. This defines an intermediate crossover regime (indicated as II in Fig. 3) between the single-channel spin-orbital Kondo phase I, where all three inter-lead processes are enhanced at low energy, and the two-channel phase IV, where these are all irrelevant.

The most pronounced suppression occurs for $\lambda_{R L}^{\perp}$, as seen on the sixth panel of Fig. 6. Indeed, in the associated flow equation (14), a prefactor $(1+\beta)^{2}$ dresses the dissipative (irrelevant) contribution $r \lambda_{R L}^{\perp}$. As a rough estimate, the coupling is completely suppressed when the total coefficient of this irrelevant term reaches $(1+\beta)^{2} r \simeq 1 / 2$. This defines the crossover line between the regions I and II, as reported in the phase diagram on Fig. [3.

Moreover, all the inter-lead couplings are completely irrelevant when $\lambda_{L R}^{\perp}$ is also driven to zero. This occurs for larger $r$ values, as seen in the fifth panel of Fig. 6, and corresponds to the condition $(1-\beta)^{2} r \simeq 1 / 2$ from the associated flow equation (14). This line separates the intermediate crossover region II from the two-channel Kondo phase IV in Fig. 3 ,

Case $\beta \lesssim 1$.

We next consider the strongly asymmetric case $\beta \lesssim 1$. From the equivalent circuit representation a large value of $\beta \lesssim 1$ implies $C_{L 1} C_{R 2} \gg C_{R 1} C_{L 2}$. A simplified parametrization for $\beta$ assuming $C_{L 1}=C_{R 2}=C$ and $C_{L 2}=C_{R 1}=\eta C$ in Eq. (15) yields

$$
\beta=\frac{1-\eta^{2}}{(1+\eta)\left(1+\eta+2 \tilde{C}_{0}\right)},
$$

with $\tilde{C}_{0}=C_{0} / C$, see Fig. 4 for a graphical illustration. A large asymmetry is hence obtained for small values of $\eta \ll 1$, in addition to a small inter-dot capacitance $C_{0}$ compared to $C$. This corresponds effectively to a situation where each dot is strongly coupled to a single lead, the upper dot to the left lead and the lower one to the right, with negligible couplings to the other lead as well as between the two dots ${ }^{37}$. Indeed, the results in Fig. 6lidentify $\lambda_{L L}^{z \uparrow}, \lambda_{L L}^{z \downarrow}$ and $\lambda_{L R}^{\perp}$ as leading couplings controlling the physical description in this region of the phase diagram. From the Hamiltonian (6) a purely orbital Kondo effect is therefore expected, where the $\uparrow$ (or $\downarrow$ ) spin configurations binds the upper dot to the left electrode (the lower dot to the right electrode respectively). Such device has been analyzed in great detail in Ref. [22] and confirms the development of an orbital Kondo effect.

Comparing the behavior of $\lambda_{L L}^{\perp}$ and $\lambda_{L R}^{\perp}$ displayed on the third an fifth panel, a symmetry under the exchange of $\beta$ in $1-\beta$ appears, related to the symmetry of the prefactors of $r$ in Eqs. (14k) and (14k) around $\beta=1 / 2$. This symmetry is also visible in the full flow of these couplings as shown on the three panels of Fig. 7 for $r=1$, associated with small, intermediate and large $\beta$ respectively. Note that for smaller values of the dissipation $r$ a weak asymmetry arises due to marginal contributions (in the RG sense) from the flow of the irrelevant coupling $\lambda \stackrel{\perp}{R}$.

The two-channel Kondo phase IV for $\beta<1 / 2$ (at $r>1 / 2$ ) is therefore replaced by an orbital Kondo phase 

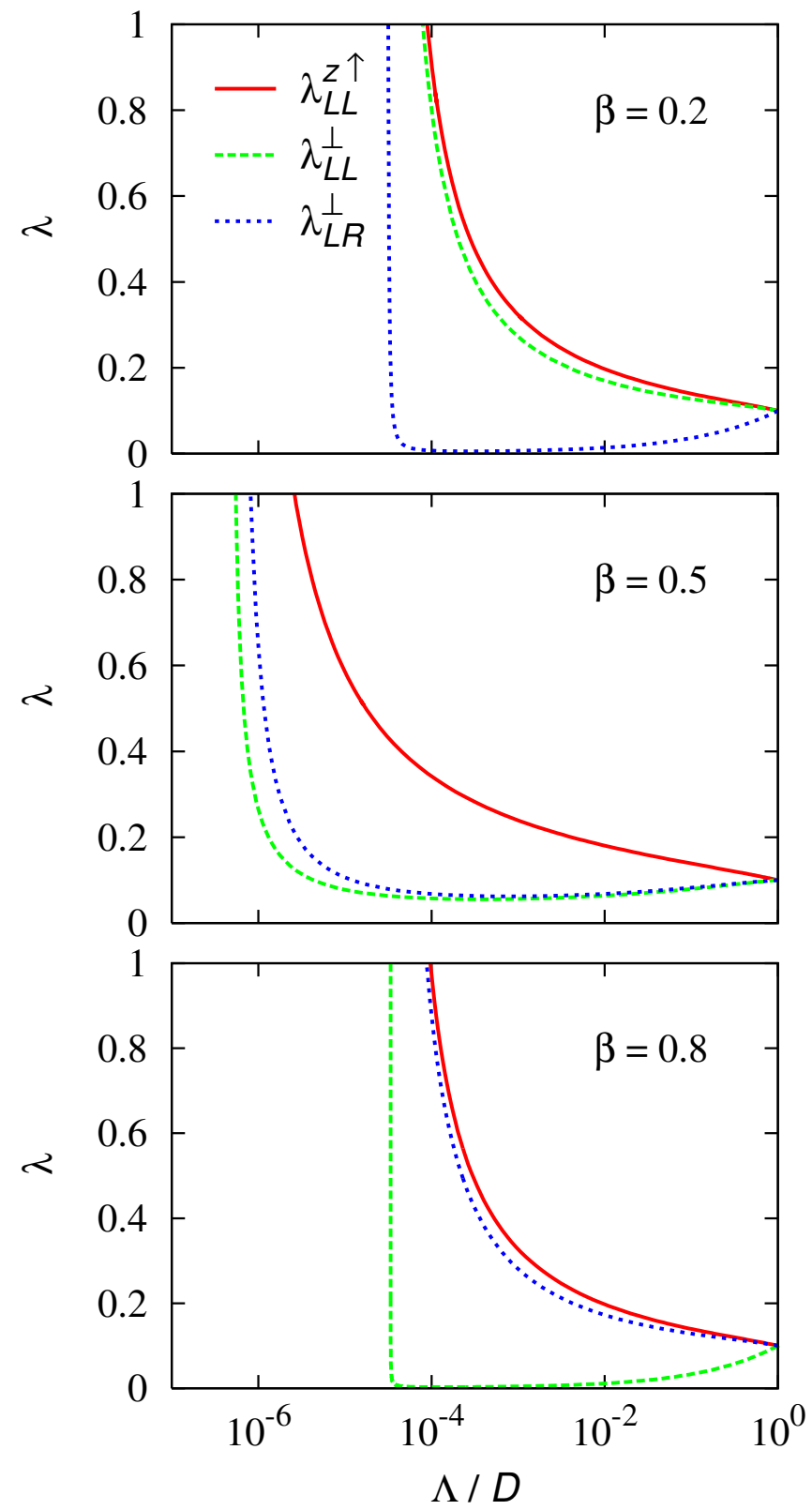

FIG. 7: (Color online) Flow of the Kondo couplings $\lambda_{L L}^{z \uparrow}$, $\lambda_{L L}^{\perp}$ and $\lambda_{L R}^{\perp}$ as a function of $\Lambda$ for a dissipation $r=1$ and asymmetry parameter $\beta=0.2,0.5,0.8$. Note the change in the Kondo scale determined by the divergence of $\lambda_{L L}^{z \uparrow}$.

for $\beta>1 / 2$, and is denoted as III in Fig. 3. The smooth boundary from II to III is given by a vanishing $\lambda_{L L}^{\perp}$ coupling, described by the condition $\beta^{2} r=1 / 2$ according to Eq. (14).).

Case $\beta \simeq 1 / 2$.

For $r>2$, all transverse couplings except $\lambda_{L L}^{z \uparrow}$ and $\lambda_{L L}^{z \downarrow}$ are driven irrelevant ${ }^{38}$. Since even now $\lambda_{L L}^{\perp}$ is driven to 0 , the spin flips, or equivalently the orbital flips, are completely suppressed and the Kondo effect disappears (formally $T_{K}=0$ ). This qualitatively different phase corresponds to a state where the impurity spin is fully localized in a given orientation by the environment. The localized phase of the model is denoted as the region $\mathrm{V}$ in Fig. 3, defined by the conditions $\beta^{2} r>1 / 2$ and $(1-$ $\beta)^{2} r>1 / 2$ from the preceding arguments. These lines correspond to a true vanishing of $T_{K}$ (from either the III and IV Kondo phases) and define a genuine quantum phase transition.

We infer by analogy with previous works on the Kondo effect in a dissipative ohmic environment that these phase transitions are of Kosterlitz-Thouless type $\stackrel{15,16,17}{1}$ For $r<2$, we recover the usual spin-orbital Kondo phase (at $r \ll 1$ ) through the intermediate crossover region II.

\section{Kondo temperature $T_{K}$}

The effect of both environmental dissipation and asymmetry in the capacitances on the Kondo temperature $T_{K}$ is reported in Fig. 8. The results for the Kondo temperature, defined by the condition $j_{L L}^{z \uparrow}\left(\Lambda=T_{K}\right)=10$, exhibit a pronounced non-monotonic behavior as a function of the asymmetry parameter $\beta$ for increasing values of the dissipation $r$. For $\beta \gtrsim 1$ the two-channel Kondo phase develops with increasing dissipation, while for $\beta \lesssim 1$ an orbital Kondo phase is observed. For intermediate values of $\beta$ and for $r>2$, a vanishing Kondo temperature indicates the appearance of the localized phase. In the more realistic case $0<r<1$, the Kondo temperature is finite, but sharply reduced for $\beta \simeq 1 / 2$.

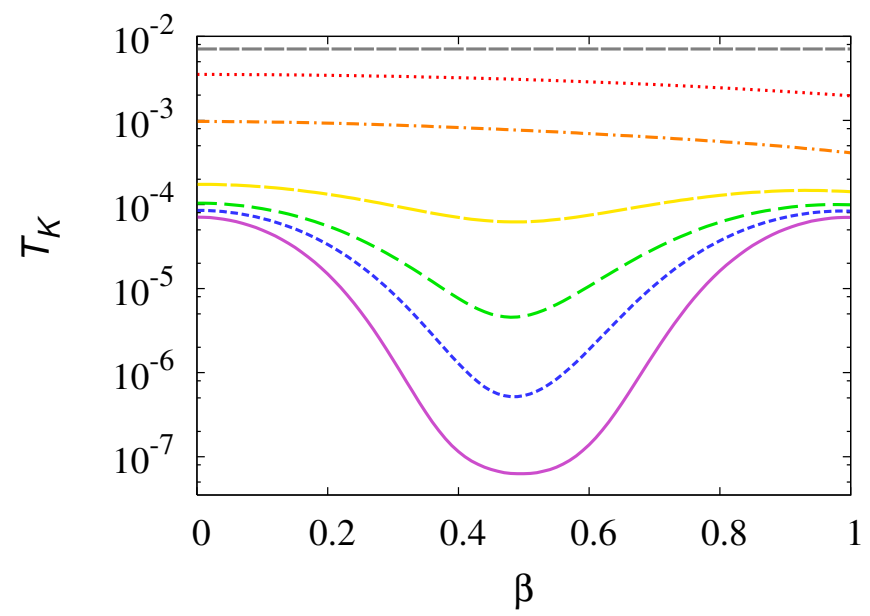

FIG. 8: (Color online) Kondo temperature $T_{K}$ as a function of asymmetry parameter $\beta$, for different values of $r=$ $0,0.1,0.25,0.5,0.75,1,1.5$ from top to bottom.

The complete evolution of the Kondo temperature in the $(r, \beta)$ phase space is provided in Fig. 9 ,

\section{Geometric effects: Asymmetric tunneling amplitudes}

We next describe the modifications to the previous phase diagram taking into account the effect of a geomet- 


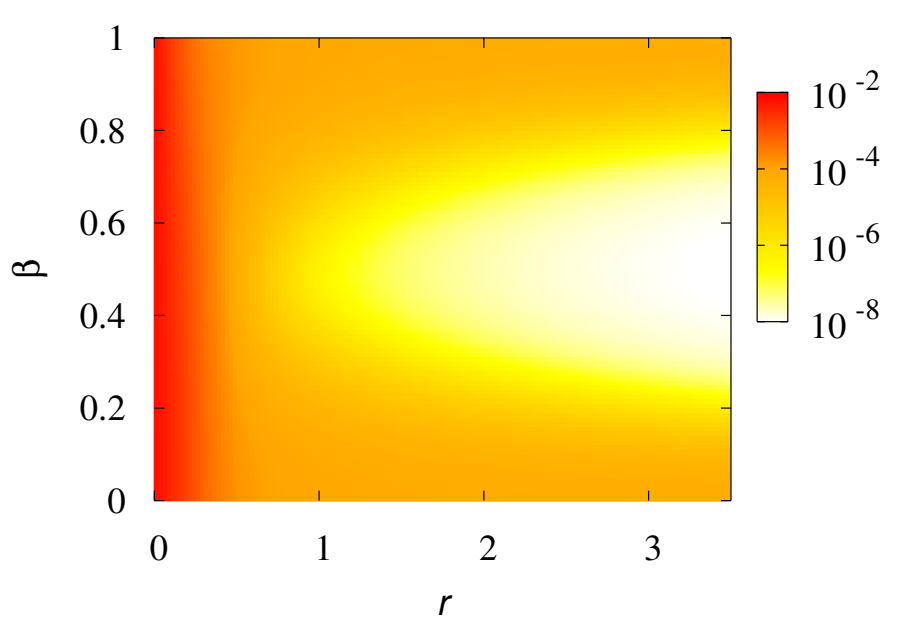

FIG. 9: (Color online) Kondo temperature $T_{K}$ as a function of dissipation $r$ and asymmetry parameter $\beta$, without geometric asymmetry in the tunneling amplitudes.

ric asymmetry both on the capacitances and on the tunneling amplitudes. In particular, the initial values of the Kondo couplings will be affected according to Eqs. (7). For the present description in terms of equivalent circuits, we will assume that the $C_{i}$ 's and $t_{i}$ 's are proportional in determining the initial values of the couplings.

In this section we will discuss the resulting phase diagram, and the corresponding behavior of the Kondo temperature. Finally, we will address the particular case of strongly asymmetric capacitances $(\beta \lesssim 1)$ corresponding to two quantum dots in series.

\section{Phase diagram and Kondo temperature $T_{K}$}

The physical properties can again be determined directly from the behavior of the different couplings during the flow. The equations describing the flow in presence of modified tunneling amplitudes due to a capacitance asymmetry are the same as in the previous treatment, Eqs. (14), whereas the initial conditions are given by Eqs. (7). For the parametrization of the tunneling amplitudes we use the model chosen above Eq. (17), with $\eta$ and $\tilde{C}_{0}$ as independent parameters. As a consequence, the maximal value of $\beta$ is limited by $\tilde{C}_{0}$. In order to access an extended range for $\beta$, we take $\tilde{C}_{0}=0.01$ in all further numerical calculations, larger values lead only to small quantitative modifications. Finally, as mentioned above, we assume that the geometric asymmetry in the tunneling amplitudes follows the same model as for the capacitances, namely: $t_{L 1}=t_{R 2}=t$ and $t_{L 2}=t_{R 1}=\eta t$. The initial (bare) Kondo couplings scale according to equation (7). Results for the solution of the flow equations Eqs. (14) with the above initial conditions are shown in Fig. 10] as a function of $\beta$ and $r$.

The symmetry $\beta \leftrightarrow 1-\beta$ for large dissipation is not preserved anymore, in particular a stronger suppression of the Kondo couplings occurs in the region $\beta>1 / 2$.

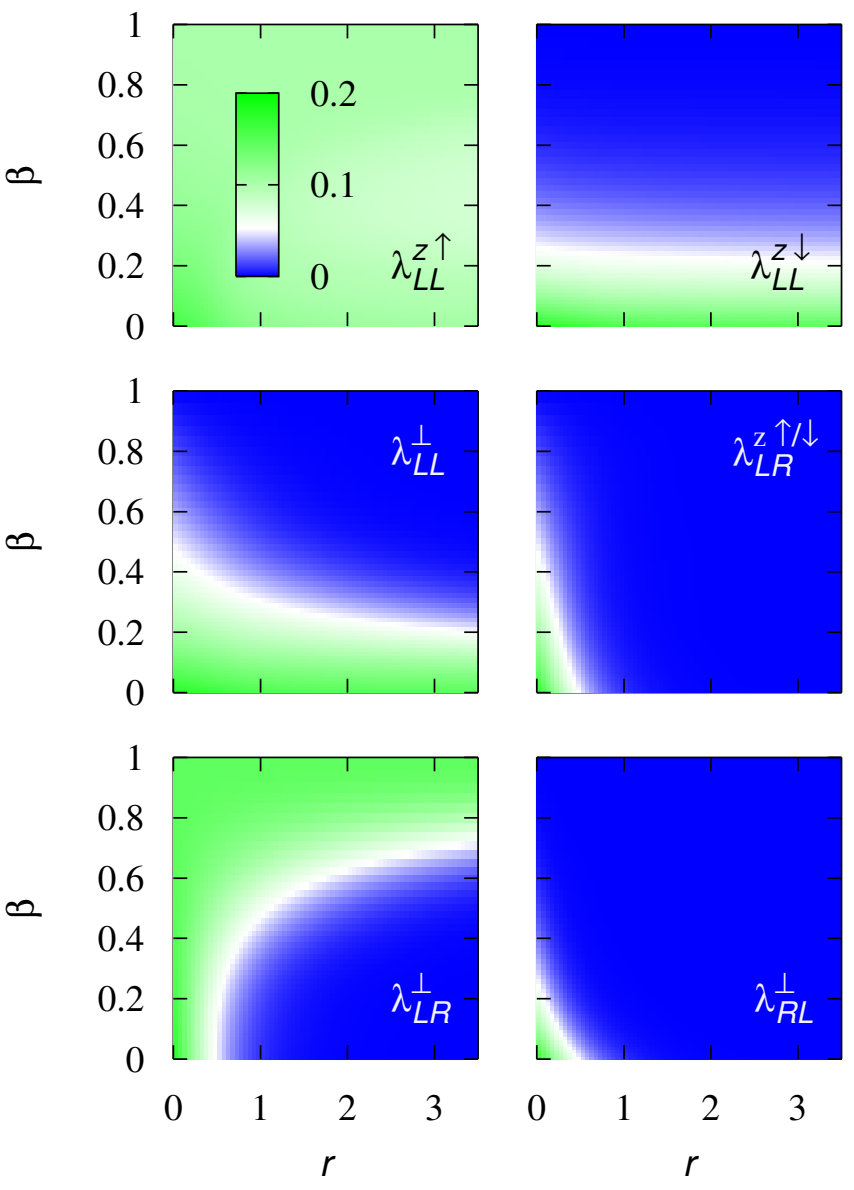

FIG. 10: (Color online) Typical value of the renormalized Kondo couplings $\lambda_{i}$ according to Eqs. (14) for the case of asymmetric capacitances and tunneling amplitudes (corresponding to asymmetric initial conditions for the Kondo couplings). These graphs are displayed as a function of dissipation $r$ and asymmetry parameter $\beta$ (with parameter $\tilde{C}_{0}=$ 0.01). The corresponding scale $\Lambda^{*}$ is determined as in Fig. 6 .

This leads to a reduction of region I in favor of regions II and III of the previous phase diagram for equal initial couplings of Fig. 3 However, the topology and qualitative properties are conserved. An analytical understanding of the flow with asymmetric initial conditions can be obtained by noting that the initial Kondo couplings (7) are simply dressed by appropriate powers of the asymmetry parameter $\eta$. A simple rescaling of the Kondo terms by these $\eta$-dependent prefactors allows to map Eqs. (14) with asymmetric couplings onto a set of effective flow equations with symmetric initial couplings and modified effective scaling dimensions. The previous analysis of the phase diagram allows to locate instantaneously the phase transition and crossover lines, that are now dressed by geometric asymmetry coefficients. These 




FIG. 11: Phase diagram as a function of dissipation $r$ and capacitance asymmetry parameter $\beta$ (assuming $\left.\tilde{C}_{0} \ll 1\right)$, including geometric asymmetry in the tunneling amplitudes. The different regions and transition lines are characterized by the same physical behavior as for symmetric tunneling amplitudes displayed in Fig. 3 .

read:

$$
\begin{aligned}
& \eta^{-2}(1+\beta)^{2} r=\frac{1}{4}\left(1+\eta^{2}\right) \\
& (1-\beta)^{2} r=\frac{1}{4}\left(1+\eta^{2}\right) \\
& \eta^{-1} \beta^{2} r=\frac{1}{4}\left(1+\eta^{2}\right),
\end{aligned}
$$

where Eq. (18 a) determines the crossover line between region I and II, Eq. (18) between region II and IV, and Eq. (18k) between region II and III. In the present case of a weak inter-dot capacitance $\tilde{C}_{0} \ll 1$, the parametrization (17) can be easily inverted as

$$
\eta=\frac{1-\beta}{1+\beta}
$$

and the use of the relation

$$
\frac{1}{2}\left(1+\eta^{2}\right)=\frac{1+\beta^{2}}{(1+\beta)^{2}}
$$

leads to a particularly simple form in terms of $\beta$ for the normalization factor appearing in Eqs. (18). The corresponding phase diagram is shown in Fig. 11 and shows only quantitative differences to the previous phase diagram Fig. 3 obtained for symmetric tunneling amplitudes. The phase diagram Fig. 11 is confirmed by the behavior of the Kondo temperature from the full numerical solution, as shown in Fig. 12 .

We finally observe that the above phase diagram is expected to be generic independently of particle-hole symmetry.

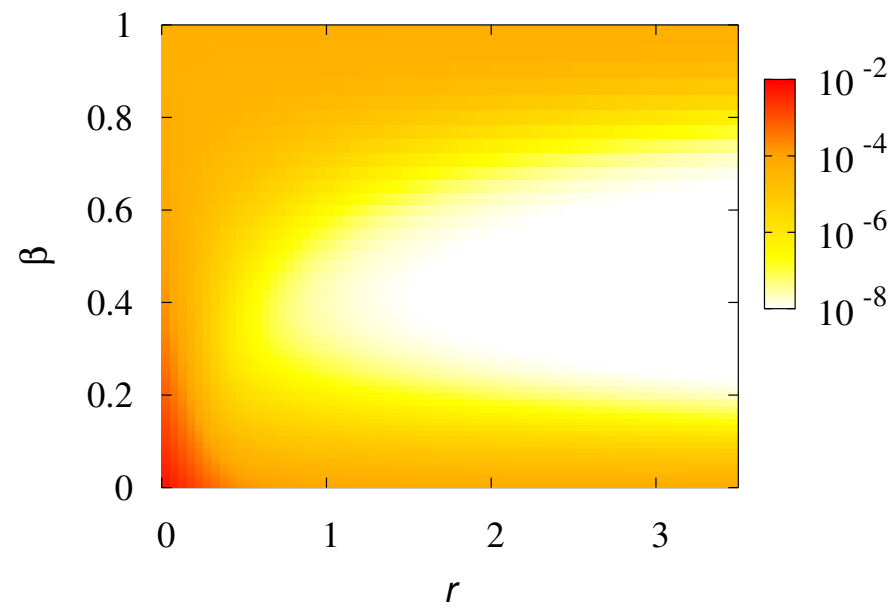

FIG. 12: (Color online) Kondo temperature $T_{K}$ as a function of dissipation $r$ and capacitance asymmetry parameter $\beta$, with geometric asymmetry in the tunneling amplitudes.

\section{A special case: serial quantum dots}

In this section, we consider the particular case of two quantum dots coupled capacitively in series as analyzed in Refs. 22, 23, 24, 25]. This corresponds to the limit $\eta=0$ in the parametrization of Fig 4 (i.e. $C_{L 2}=C_{R 1}=0$ ) and to $t_{L 2}=t_{R 1}=0$ : it leads therefore to a maximal asymmetry in the capacitances and tunneling amplitudes. A schematic representation of the resulting serial quantum dot setup is shown in Fig. 13.

The equations describing the flow of the remaining couplings $\lambda_{L L}$ and $\lambda_{L R}$, from the equations for $\lambda_{L L}^{z \uparrow}$ and $\lambda_{L R}^{\perp}$ of system (14), read

$$
\begin{aligned}
& \frac{d \lambda_{L L}}{d \ln \Lambda}=-\frac{1}{2}\left[\left(\lambda_{L L}\right)^{2}+\left(\lambda_{L R}\right)^{2}\right] \\
& \frac{d \lambda_{L R}}{d \ln \Lambda}=(1-\beta)^{2} r \lambda_{L R}-\lambda_{L R} \lambda_{L L},
\end{aligned}
$$

where the capacitance asymmetry parameter reduces to $\beta=1 /\left(1+2 \tilde{C}_{0}\right)$. The redefinition $\Lambda \rightarrow \Lambda^{2}$ and $2(1-\beta)^{2} r \rightarrow r$ in the above equations reduces to the system (16), that describes the flow of the inter- and intralead processes for a single quantum dot in an ohmic environment. The $(r, \beta)$ phase diagram presents therefore two regimes, separated by the condition $2(1-\beta)^{2} r=1 / 2$. The regions I, II and III of Fig. 3 correspond to a single-

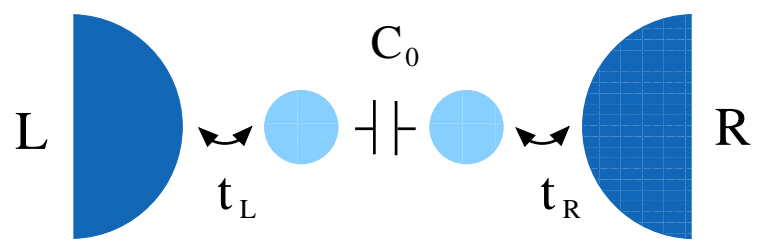

FIG. 13: (Color online) Schematic representation of a serial DD corresponding to the setup proposed in Fig. 1 in the limit of maximal tunneling and capacitance asymmetry. 
channel Kondo regime, while regions IV and V to a twochannel Kondo regimes.

In addition, the respective Kondo temperature appears lowered by $T_{K} \rightarrow\left(T_{K}\right)^{2}$ with respect to the single dot.

\section{CONCLUSION AND DISCUSSION}

The present analysis extends the recent investigation of Ref. 21] of the effects of electromagnetic noise on the Kondo effect in a quantum dot to a capacitively coupled DD device, characterized by entangled spin and orbital degrees of freedom. The low-energy physics of the above spin-orbital Kondo model in presence of an ohmic environment of resistance $R$ exhibits a rich phase diagram as a function of the capacitance asymmetry parameter $\beta$ and dimensionless resistance $r=R / R_{K}$, with the quantum value $R_{K}=h / e^{2}$. Using a perturbative renormalization-group approach, we could identify several crossover regions between different Kondo fixed points and a distinctive localized phase (where spin-flip processes are fully suppressed) for large values of $r$ and $\beta \simeq 1 / 2$. The corresponding Kondo temperature displays a pronounced non-monotonic behavior with $\beta$, with a strong reduction in proximity of the localized phase. Additional geometric asymmetries in the tunneling amplitudes further reduce spin-flip processes, thus favoring the development of the localized phase.

Finally, the present results lead to interesting consequences in the context of quantum information theory, in particular on the efficiency of spin filtering devices in presence of environmental fluctuations. Promising proposals for a controlled realization of spin qubits and on their operations rely on the use of entangled spin and orbital degrees of freedom. In view of an experimental realization, an essential issue concerns the spin coherence. The present work helps to understand how the phase and spin-coherent transmission through the device is affected by inelastic interaction with the environment. To minimize those effects, the device should be as symmetric as possible, in its geometry, and operate at low bias voltage. The combination of recent experimental realizations of both strongly capacitive $\operatorname{dots}^{35}$ and a strongly resistive environment $\underline{36}$ represents a promising prospect.

\section{Acknowledgments}

This work has been supported by the contract ANR_05_NANO_050_S2.

\section{APPENDIX A: COMPLETE SET OF RG EQUATIONS}

The RG flow equations of the ten Kondo couplings are derived in straightforward manner paying attention to the fact that most of them acquire some anomalous dimensions. At second order in $\lambda$, the RG flow reads

$$
\begin{aligned}
& \frac{d \lambda_{L L}^{z \uparrow}}{d \ln \lambda}=-\frac{1}{2}\left[\left(\lambda_{L L}^{z \uparrow}\right)^{2}+\left(\lambda_{L L}^{\perp}\right)^{2}+\left(\lambda_{L R}^{z \uparrow}\right)^{2}+\left(\lambda_{L R}^{\perp}\right)^{2}\right] \\
& \frac{d \lambda_{L L}^{z \downarrow}}{d \ln \lambda}=-\frac{1}{2}\left[\left(\lambda_{L L}^{z \downarrow}\right)^{2}+\left(\lambda_{L L}^{\perp}\right)^{2}+\left(\lambda_{L R}^{z \downarrow}\right)^{2}+\left(\lambda_{R L}^{\perp}\right)^{2}\right] \\
& \frac{d \lambda_{L L}^{\perp}}{d \ln \lambda}=\left(\kappa_{L 2}-\kappa_{L 1}\right)^{2} r \lambda_{L L}^{\perp}-\frac{1}{2}\left[\lambda_{L L}^{\perp}\left(\lambda_{L L}^{z \uparrow}+\lambda_{L L}^{z \downarrow}\right)+\lambda_{L R}^{\perp} \lambda_{L R}^{z \downarrow}+\lambda_{R L}^{\perp} \lambda_{L R}^{z \uparrow}\right] \\
& \frac{d \lambda_{L R}^{z \uparrow}}{d \ln \lambda}=\left(\kappa_{L 1}+\kappa_{R 1}\right)^{2} r \lambda_{L R}^{z \uparrow}-\frac{1}{2}\left[\lambda_{L R}^{z \uparrow}\left(\lambda_{L L}^{z \uparrow}+\lambda_{R R}^{z \uparrow}\right)+\lambda_{L L}^{\perp} \lambda_{R L}^{\perp}+\lambda_{R R}^{\perp} \lambda_{L R}^{\perp}\right] \\
& \frac{d \lambda_{L R}^{z \downarrow}}{d \ln \lambda}=\left(\kappa_{L 2}+\kappa_{R 2}\right)^{2} r \lambda_{L R}^{z \downarrow}-\frac{1}{2}\left[\lambda_{L R}^{z \downarrow}\left(\lambda_{L L}^{z \downarrow}+\lambda_{R R}^{z \downarrow}\right)+\lambda_{L L}^{\perp} \lambda_{L R}^{\perp}+\lambda_{R R}^{\perp} \lambda_{R L}^{\perp}\right] \\
& \frac{d \lambda_{L R}^{\perp}}{d \ln \lambda}=\left(\kappa_{L 1}+\kappa_{R 2}\right)^{2} r \lambda_{L R}^{\perp}-\frac{1}{2}\left[\lambda_{L R}^{\perp}\left(\lambda_{L L}^{z \uparrow}+\lambda_{R R}^{z \downarrow}\right)+\lambda_{L L}^{\perp} \lambda_{L R}^{z \downarrow}+\lambda_{R R}^{\perp} \lambda_{L R}^{z \uparrow}\right] \\
& \frac{d \lambda_{R}^{\perp}}{d \ln \lambda}=\left(\kappa_{R 1}+\kappa_{L 2}\right)^{2} r \lambda_{R L}^{\perp}-\frac{1}{2}\left[\lambda_{R L}^{\perp}\left(\lambda_{L L}^{z \downarrow}+\lambda_{R R}^{z \uparrow}\right)+\lambda_{L L}^{\perp} \lambda_{L R}^{z \uparrow}+\lambda_{R R}^{\perp} \lambda_{L R}^{z \downarrow}\right],
\end{aligned}
$$

where the parameter $r=R / R_{K}$ defines the normalized resistance. The RG equations for the Kondo couplings $\lambda_{R R}^{z \uparrow / \downarrow, \perp}$ are simply inferred from $\lambda_{L L}^{z \uparrow / \downarrow, \perp}$ by exchanging $L \leftrightarrow R$. Though $\lambda_{\gamma \gamma}^{z \uparrow}$ may be equal to $\lambda_{\gamma \gamma}^{z \downarrow}$ at the bare level, some asymmetry may be generated through the renormalization of the couplings $J_{L R}^{z \uparrow / \downarrow}$. Similarly, a finite prefactor $\left(\kappa_{L 2}-\kappa_{L 1}\right)^{2}$ of $r$ in Eq. (A1c) can arise dynamically in the flow even for equal initial conditions, leading to a suppression of the orthogonal coupling $\lambda_{L L}^{\perp}$. The disappearance of the Kondo effect in this situation 
leads to a qualitatively different picture as in the noisy single-dot setup discussed previously. ${ }^{21}$

An important question concerns the effect of the frequency dependence of the circuit's impedance, neglected in the present treatment. The full energy distribution function $P(E)$ of the environmental modes for an ohmic dissipation can be taken into account within the $P(E)$ theory. For a single quantum dot of Sec. IVB 2, the main modification of the flow equations (16) consists in the vanishing of the dissipative term appearing in Eq. (16b) above an energy cutoff $\omega_{c} \simeq 1 /(R C)$, where $R$ is the resistance of the environment and $C$ the effective capacitance of the equivalent circuit. The absence of $\mathrm{DCB}$ for $\Lambda>\omega_{c}$ leads to an initial renormalization of all couplings without environmental effects; for $\Lambda<\omega_{c}$ the dissipative lowenergy behavior comes into play. Concerning the associated Kondo temperature, in addition to the dissipation effects a sensitive dependence on the full spectral function of excited environmental modes is observed. In particular, the onset of DCB at scale $\omega_{c}$ implies in general a less pronounced decrease of the associated Kondo temperature for increasing dissipation than previously predicted. For a more detailed analysis we refer to Ref. [21].
1 D. Loss and D.P. DiVincenzo, Phys. Rev. A 57, 120 (1998).

2 E.A. Laird, J.R. Petta, A.C. Johnson, C.M. Marcus, A. Yacoby, M.P. Hanson, and A.C. Gossard, Phys. Rev. Lett. 97, 056801 (2006); F.H.L. Koppens, C. Buizert, K.J. Tielrooij, I.T. Vink, K.C. Nowack, T. Meunier, L.P. Kouwenhoven, and L.M.K. Vandersypen, Nature 442, 766 (2006).

3 P. Recher, E.V. Sukhorukov, and D. Loss, Phys. Rev. B 63, 165314 (2001); O. Sauret, T. Martin, and D. Feinberg, Phys. Rev. B 72, 024544 (2005).

4 O. Sauret, D. Feinberg, and T. Martin, Phys. Rev. B 69, 035332 (2004).

5 A. Bychkov and E.I. Rashba, J. Phys. C 17 (2002).

6 T. Koga, J. Nitta, T. Akazaki, and H. Takayanagi, Phys. Rev. Lett. 89, 046801 (2002).

7 T. Koga, J. Nitta, and M. van Veenhuizen, Phys. Rev. B 70, 161302 (2004); T. Koga, Y. Sekine, and J. Nitta, Phys. Rev. B 74, 041302(R) (2006).

8 D. Feinberg and P. Simon, Appl. Phys. Lett. 85, 1846 (2004).

9 P. Simon and D. Feinberg, Phys. Rev. Lett. 97, 247207 (2006).

10 G.-L. Ingold and Yu. V. Nazarov, in "Single Charge Tunneling", edited by H. Grabert and M. H. Devoret, NATO ASI Series B, Vol. 294, pp. 21-107 (Plenum Press, New York, 1992).

11 M. H. Devoret and H. Grabert, in "Single Charge Tunneling", edited by H. Grabert and M. H. Devoret, NATO ASI Series B, Vol. 294, pp. 1-19 (Plenum Press, New York, 1992).

12 R. Aguado and L.P. Kouwenhoven, Phys. Rev. Lett. 84, 1986 (2000).

13 F. Marquardt and C. Bruder, Phys. Rev. B 68, 195305 (2003).

14 E. Dupont and K. Le Hur, Phys. Rev. B 73045325 (2006).

15 K. Le Hur, Phys. Rev. Lett. 92, 196804 (2004).

16 M.-R. Li, K. Le Hur, and W. Hofstetter, Phys. Rev. Lett. 95, 086406 (2005).

17 L. Borda, G. Zarand, and P. Simon, Phys. Rev. B 72, 155311 (2005).

18 L. Borda, G. Zarand, and D. Goldhaber-Gordon, cond-mat/0602019.

19 A. Kaminski, Yu.V. Nazarov, and L.I. Glazman, Phys. Rev. B 62, 8154 (2000).

20 R. López, R. Aguado, G. Platero, and C. Tejedor, Phys. Rev. B 64, 075319 (2001).

21 S. Florens, P. Simon, S. Andergassen, and D. Feinberg,
Phys. Rev. B 75, 155321 (2007).

${ }^{22}$ L. Borda, G. Zarand, W. Hofstetter, B.I. Halperin, and J. von Delft, Phys. Rev. Lett. 90, 026602 (2003).

23 T. Pohjola, H. Schoeller, and G. Schön, Europhys. Lett. 54, 241 (2001).

24 M.R. Galpin, D.E. Logan, and H.R. Krishnamurthy, Phys. Rev. Lett. 94, 186406 (2005); ibid, J. Phys.: Condens. Matter 18, 6545 (2006).

25 M.-R. Li and K. Le Hur, Phys. Rev. Lett. 93, 176802 (2004).

26 P. Recher, E.V. Sukhorukov, and D. Loss, Phys. Rev. Lett. 85, 1962 (2000).

27 R. Hanson, L.H.Willems vanBeveren, I.T. Vink, J.M. Elzerman, W.J.M. Naber, F.H.L. Koppens, L.P. Kouwenhoven, and L.M.K. Vandersypen, Phys. Rev. Lett. 94, 196802 (2005).

${ }^{28}$ K. Le Hur and P. Simon, Phys. Rev. B 67, 201308(R) (2003); K. Le Hur, P. Simon, and L. Borda, Phys. Rev. B 69, 045326 (2004); K. Le Hur, P. Simon, and D. Loss, Phys. Rev. B 75, 035332 (2007).

29 P. Jarillo-Herrero, J. Kong, H. S. J. van der Zant, C. Dekker, L. P. Kouwenhoven, and S. De Franceschi, Nature 434, 484 (2005).

30 M.-S. Choi, R. Lopez, and R. Aguado, Phys. Rev. Lett. 95, 067204 (2005).

31 J. Martinek, Y. Utsumi, H. Imamura, J. Barnas, S. Maekawa, J. König, and G. Schön, Phys. Rev. Lett. 91, 127203 (2003); M.-S. Choi, D. Sanchez and R. Lopez, Phys. Rev. Lett. 92, 056601 (2004); P. Simon, P.S. Cornaglia, D. Feinberg, and C.A. Balseiro, Phys. Rev. B 75, 045310 (2007).

32 H. Grabert, G.-L. Ingold, M.H. Devoret, D. Estéve, H. Pothier, and C. Urbina, Z. Phys. B 84, 143 (1991); G.-L. Ingold, P. Wyrowski, and H. Grabert, Z. Phys. B 85, 443 (1991).

33 A. A. Odintsov, V. Bubanja, and G. Schön, Phys. Rev. B 46, 6875 (1992).

34 U. Geigenmüller and Yu.V. Nazarov, Phys. Rev. B 44, 10953 (1991).

35 A. Hübel, J. Weiss, W. Dietsche, and K. v. Klitzing, App. Phys. Lett. 91, 102101 (2007).

36 C. Altimiras, U. Gennser, A. Cavanna, D. Mailly, and F. Pierre, to be published in Phys.Rev. Lett. (2007).

37 Notice that the orbital Kondo effect requires a minimum coupling $\tilde{C}_{0}$, in order to get a large enough effective charging energy $E_{c}$. 
38 Note that the divergence of $\lambda_{L L}^{z \uparrow}$ in the localized region is unphysical and is due to the perturbative treatment. 